\title{
The transfer of heat in turbulent boundary layers with injection or suction: universal laws and Stanton number equations
}

\author{
Marcello A. Faraco-Medeiros and Atila P. Silva-Freire \\ Laboratório de Mecânica dos Fluidos/Aerodinâmica, Programa de Engenharia Mecânica \\ (COPPE/UFRJ), C.P. 68503, 21945-Rio de Janeiro, Brazil
}

(Received 14 February 1991 and in final form 17 May 1991)

\section{INTRODUCTION}

THE INJECTION or suction of fluid into the turbulent boundary layer through a porous surface is an effective means of promoting the thermal protection of walls $[1,2]$. The transpired fluid convects thermal energy away from the wall, improving the ability of surfaces to withstand a high-temperature environment. Despite this classical application, transpired turbulent boundary layers have also been extensively studied in connection with a variety of other applications. For example, injection or suction of fluid can be effectively used to reduce the drag of flows around bodies [3-5]. Also, variations of the transpiration problem are found when the injected fluid is a chemical reagent or when either evaporation or sublimation occurs.

For low speed flows, a good account of the transpiration phenomenon is given in the literature by a number of analyses of the problem [3-14]. Studies of the velocity boundary layer have established solid expressions for the law of the wall and for the law of the wake $[3,5]$. These expressions have been derived for a range of flow conditions, yielding a bilogarithmic expression for the skin friction. This skin friction equation is much less sensitive than the momentum-integral equation to small changes in the flow parameters and so gives much more reliable results [8]. Unfortunately, for the thermal turbulent boundary layer with transpiration, no equivalent equation has been derived for predictions of the friction temperature. Most of the work has been conducted at Stanford University $[1,2,6,7]$ to the study of universal correlations for the temperature profile and for the prediction of Stanton number. These studies, together with the analyses of refs. [9-11], show that close to the wall the temperature profile has, as predicted by simple theoretical mixing-length based approaches, a logarithmic behaviour. In this case, the similarity parameters and the constants in the expressions must be such that their dependence on the Prandtl number is adequately taken into account [11].

This paper derives two new expressions for the law of the wall and shows how they can be extended to the defect layer simply by using Coles' function. The resulting temperature defect expressions yield bilogarithmic expressions for Stanton number similar to the skin friction equation which differ markedly from the previously proposed expressions and are hence presented here for the first time. The Stanton number has normally been evaluated by applying a correction factor to the unblown Stanton number, $S t_{0}$, according to an expression derived by Spalding [12] in the sixties. The present formulation advances this formulation since it incorporates all the advantages of the skin friction equation [8], besides dispensing the previous knowledge of $\boldsymbol{S t}_{0}$. The expression for Stanton, however, is sensitive to the flow hydrodynamics through $u_{\tau}$ as well as to the transfer of heat through $t_{z}$ [2]. This means that inaccuracies in the prediction of $u_{\mathrm{r}}$ and $t_{\tau}$ are immediately propagated into $S t$ in a cumulative manner. To avoid this difficulty we then propose an alternative approach where $S t$ can be evaluated independently from the knowledge of $u_{\mathrm{r}}$.
The analysis is carried out for two-dimensional, steady flows over aerodynamically smooth surfaces with no external pressure gradients. Extension of the present results to more complex flows will be published shortly.

\section{THE THERMAL TURBULENT BOUNDARY LAYER ON A POROUS SURFACE}

To study the effects of the transpiration on the boundary layer we divide the flow region into distinct parts where certain dominant effects can be used to derive simplified equations. The formulation of the transpiration problem basically differs from the solid surface problem in the sense that the inertia effects near the wall can no longer be neglected $[1,5,8]$. Therefore, for the near-wall dominated part of the flow, the approximate energy equation becomes

$$
\frac{\partial Q^{\prime \prime}}{\partial y}=\rho c_{p} v_{n} \frac{\partial t}{\partial y}
$$

This equation, together with the velocity solution [6], the mixing-length hypothesis

$$
Q^{\prime \prime}=\rho c_{p} k_{m} k_{t} y^{2} \frac{\partial u}{\partial y} \frac{\partial t}{\partial y}
$$

and the boundary condition

$$
Q^{\prime \prime}(0)=Q_{w}^{\prime \prime}=\rho c_{p} u_{r} t_{r}
$$

yields

$$
\begin{array}{r}
\frac{k_{m}}{k_{i}} \frac{2}{v_{w}^{+}\left\{\left(\frac{v_{w}^{+} t^{+}+1}{v_{w}^{+} t_{b}^{+}+1}\right)^{k / 2 k_{m}}\right.}\left[\frac{v_{w}^{+}}{2}\left(\frac{1}{k_{m}} \ln \frac{y_{a}^{+}}{y_{b}^{+} \operatorname{Pr}}\right)+\sqrt{ }\left(1+v_{w}^{+} u_{a}^{+}\right)\right] \\
\left.-\sqrt{ }\left(1+v_{w}^{+} u_{a}^{+}\right)\right\}=\frac{1}{k_{t}} \ln \frac{y^{+} \operatorname{Pr}}{y_{b}^{+} \operatorname{Pr}}
\end{array}
$$

where $v_{w^{+}}^{+}$denotes $V_{\mathrm{v}} / u_{\mathrm{r}}$ and the pair $\left(y_{b}^{+}, u_{b}^{+}\right)$is a constant of integration which must be determined experimentally. Parameters $k_{m}$ and $k_{t}$, characteristic of the turbulence modelling, must also be determined experimentally. The above equation is the so-called law of the wall; it is here cast for the first time in this form.

An analysis of the data of refs. $[5,6]$ shows that the value of $t^{+}$where the laminar-conductive and the turbulent solutions meet is nearly independent of the injection rate. Calling this value $t_{b}^{+}$, it can be determined by patching the laminar and the fully turbulent layer solutions for unblown flows. This results in

$$
t_{b}^{+}=10 \text {. }
$$

Parameter $y_{b}^{+}$, obtained through the laminar-conductive layer solution

$$
t^{+}=\frac{1}{v_{w}^{+}}\left(\mathrm{e}^{\left(t_{u}^{+} r^{+} p_{r l}\right.}-1\right)
$$




\section{NOMENCLATURE}

$A, B, B^{\prime}$ parameter in laws of the wall

$C f$ skin friction coefficient

$c_{p} \quad$ specific heat at constant pressure

$k_{m} \quad$ von Karman's constant in velocity profile

$k_{i}, k_{i}^{\prime}$ von Karman's constants in temperature profiles

$\operatorname{Pr} \quad$ Prandtl number

$Q^{\prime \prime} \quad$ heat flux

Re Reynolds number

St Stanton number

$T$ temperature

$t$ dimensionless temperature,

$\left(T-T_{w^{\prime}}\right) /\left(T_{\times}-T_{\mathrm{u}}\right)$

$t_{\tau} \quad$ friction temperature

$t^{+}, t^{*}$ inner layer similarity temperatures,

$\left(T-T_{11}\right) / t_{t}, t / \sqrt{ }(S t)$

$U, V$ velocity components

$u_{\tau} \quad$ friction velocity

$v_{4} \quad V_{i} ; l^{\prime}$

$u^{+}, l_{n}^{+}, v^{*} \quad U i u_{\tau}, V_{1, i}^{i} u_{\tau}, V_{x} / U, v^{i}(S t)$

w Coles function $y^{+}, y^{*}$ inner layer similarity coordinates, $y u_{\mathrm{r}} / \nu_{\mathrm{n}}$ $v i \times v(S t) i t$

Greek symbols

$\alpha \quad$ thermal diffusivity

$\delta_{m}, \delta_{t} \quad$ velocity boundary layer thickness, temperature boundary layer thickness

$\Delta \quad$ enthalpy thickness

$\theta$ momentum thickness

$v \quad$ kinetic viscosity

$\Pi_{t}, \Pi_{i}^{\prime}$ temperature wake profiles

$\rho$ density

$\tau \quad$ shear stress.

Subscripts

$\begin{array}{ll}a, b & \text { constants of integration } \\ m & \text { velocity } \\ t & \text { temperature } \\ w & \text { conditions at wall } \\ \infty & \text { external flow conditions. }\end{array}$

is given by

$$
y_{h}^{+}=\frac{\ln v_{1}^{+} t_{h}^{+}+1}{r_{u}^{+} \operatorname{Pr}} .
$$

Of course, in the limit as $v_{w} \rightarrow 0$, equation (4) reduces to the solid surface solution. Results provided by equation (4) are compared with the experimental data of ref. [6] in Fig. 1. As can be seen, the agreement is good for the blowing data. The discrepancies found for the suction data were expected since in the experiments suction was applied over a long stretch of porous surface and, under this condition, the turbulent fluctuations in the boundary layer were partially or even completely removed, resulting in a not very well established turbulent boundary layer. Please note the very low values of $R e$ for the suction data. It is worth stressing here that the temperature data agree much better with the present theory (equation (4)) than the corresponding velocity data agree with the equation derived by Simpson [5] if classical values of $k_{n}(0.41)$ and $A(5.0)$ are used to correlate the data.

Equation (4) can more generally be written as

$$
F_{t}\left(V_{n},\left(T-T_{n}\right), u_{2}, l_{\tau}\right)=f_{i}\left(y^{+}, P r\right) .
$$

An extension of this expression to the defect layer can be obtained if we follow Stevenson [4] and make

$F_{i}\left(V_{n,},\left(T-T_{11}\right), u_{\tau}, t_{\imath}\right)$

$$
-F_{m}\left(V_{n},\left(T_{,}, T_{n}\right), u_{\tau}, t_{\tau}\right)=g_{i}\left(\frac{y}{\delta_{l}}\right) .
$$

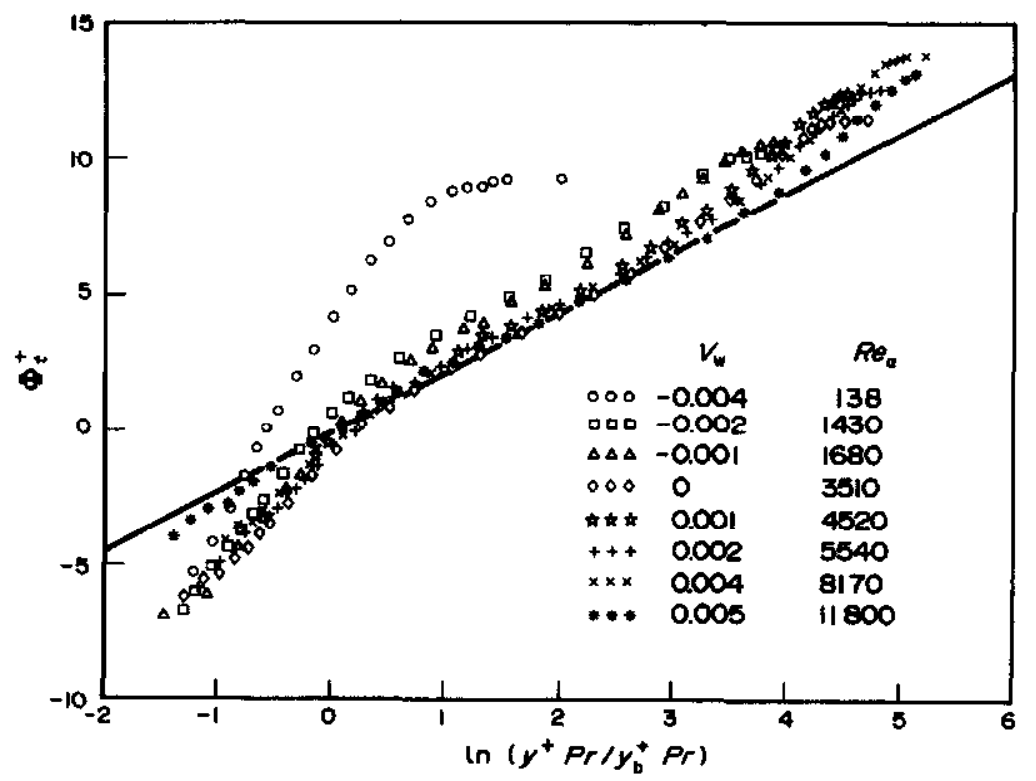

FIG. 1. Temperature law of the wall for transpired flow. Data from Whitten et al. [6]. 
Combination of equations ( 8 ) and (9) yields

$$
\begin{gathered}
\frac{2}{v_{w}^{+}}\left\{\left(\frac{v_{w}^{+} t^{+}+1}{v_{w}^{+} t_{b}^{+}+1}\right)^{k_{t} / 2 k_{m}}\left[\frac{v_{w}^{+}}{2}\left(\frac{1}{k_{m}} \ln \frac{y_{a}^{+}}{y_{b}^{+} \operatorname{Pr}}\right)+\sqrt{ }\left(1+v_{w}^{+} u_{a}^{+}\right)\right]\right. \\
\left.-\sqrt{ }\left(1+v_{w}^{+} u_{a}^{+}\right)\right\}=\frac{1}{k_{m}} \ln \frac{y^{+} \operatorname{Pr}}{y_{b}^{+} \operatorname{Pr}_{r}}+\frac{\Pi_{t}}{k_{m}} w\left(\frac{y}{\delta_{t}}\right) .
\end{gathered}
$$

Results obtained with the above formulation are shown in Fig. 2.

An equation for the prediction of the friction temperature is readily obtained if we substitute $(y, T)=\left(\delta_{2}, T_{\infty}\right)$ into equation (10) and obtain

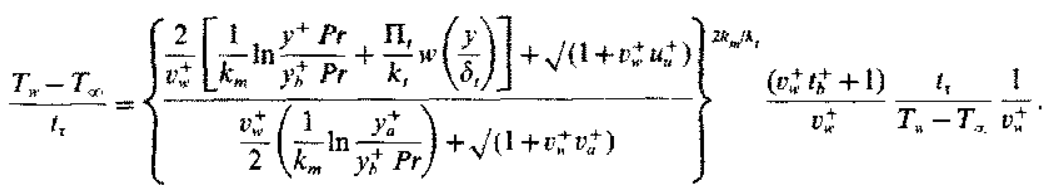

obtained by adding Coles' function to its right-hand side. Solutions with the alternative approach are shown in Figs. 3 and 4 together with the data of ref. [6].

For transpired flow, the Stanton equation can be written as

$$
\begin{aligned}
1= & \sqrt{ }(S t)\left(\frac{1}{k_{s}^{\prime}} \ln \frac{\delta_{t} U_{\infty} \sqrt{ }(S t) \operatorname{Pr}}{v y_{b}^{*} P r}+\frac{2 \Pi_{t}^{\prime}}{k_{t}^{\prime}}\right)^{2} \frac{v_{w}^{*}}{4}+\sqrt{ }(S t) \\
& \times\left[\left(\frac{1}{k_{t}^{\prime}} \ln \frac{\delta_{t} U_{\infty} \sqrt{ }(S t) P r}{v y_{b}^{*} P r} *+\frac{2 \Pi_{t}^{\prime}}{k_{t}^{\prime}}\right) \sqrt{ }\left(1+t_{b}^{*} v_{w}^{*}\right)+t_{b}^{*}\right] .
\end{aligned}
$$

The problem can be given an alternative solution if we consider now the similarity variables defined by

$$
t^{*}=\frac{T-T_{w}}{\left(T_{x}-T_{w}\right) \sqrt{ }(S t)}, y^{*}=\frac{y U_{\infty} \sqrt{ }(S t)}{v} .
$$

These similarity parameters were first suggested by Blackwell et al. [7]. They present the advantage of not including $u_{s}$ in their definitions so that $S t$ can be immediately evaluated, by the Stanton equation, from the main flow conditions. Then assuming that the velocity fluctuations, $u / U_{\infty}$, are proportional to the temperature fluctuations, $t^{\prime} / \Delta T$. so that the mixing-length equation can be written as

$$
\tau_{v t}=k_{t}^{\prime 2} y^{2}\left(\frac{\partial t}{\partial y}\right)^{2}
$$

it follows that the law of the wall, equation (4), becomes

$$
\frac{2}{v_{w}^{*}}\left[\sqrt{ }\left(1+t^{*} v_{w}^{*}\right)-\sqrt{ }\left(1+t_{b}^{*} v_{w}^{*}\right)\right]=\frac{1}{k_{t}^{\prime}} \ln \frac{y^{*} P r}{y_{b}^{*} \operatorname{Pr}}
$$

clearly a much simpler equation. Here we have $v_{w}^{*}=v_{w}$ l $U_{x, V^{\prime}}(S t)$.

Extension of equation (13) to the defect region is again
We also observed that the temperature wake profile varies with the flow conditions, as does the velocity wake profile. Indeed, we know that $\Pi_{m}$ varies with $R e_{\theta}$, its value departing from 0.0 and asymptotically approaching 0.55 . Here we note that $\Pi_{s}$ and $\Pi_{\text {, }}$, bave the same qualitative behaviour of $\Pi_{\mathrm{w}}$ (see Figs. 5 and 6$)$, varying asymptotically with $R e_{\Delta}(\Delta=$ enthalpy thickness of the boundary layer). Both curves for $\Pi_{\text {, and }} \Pi_{i}^{\prime}$ suggest the asymptotic values to which these two parameters should tend. An insufficient number of experimental data, however, does not allow us to make a definitive assertion about these values.

Finally, predictions of Stanton number for several injection rates are tested against the data of Whitten et al. [6] in Table 1. This table also presents predictions obtained through the Spalding formulation [12], which yields

$$
\frac{S t}{S t_{0}}=\frac{\ln \left(1+\frac{v_{n}}{S t}\right)}{\frac{v_{w}}{S t}}
$$

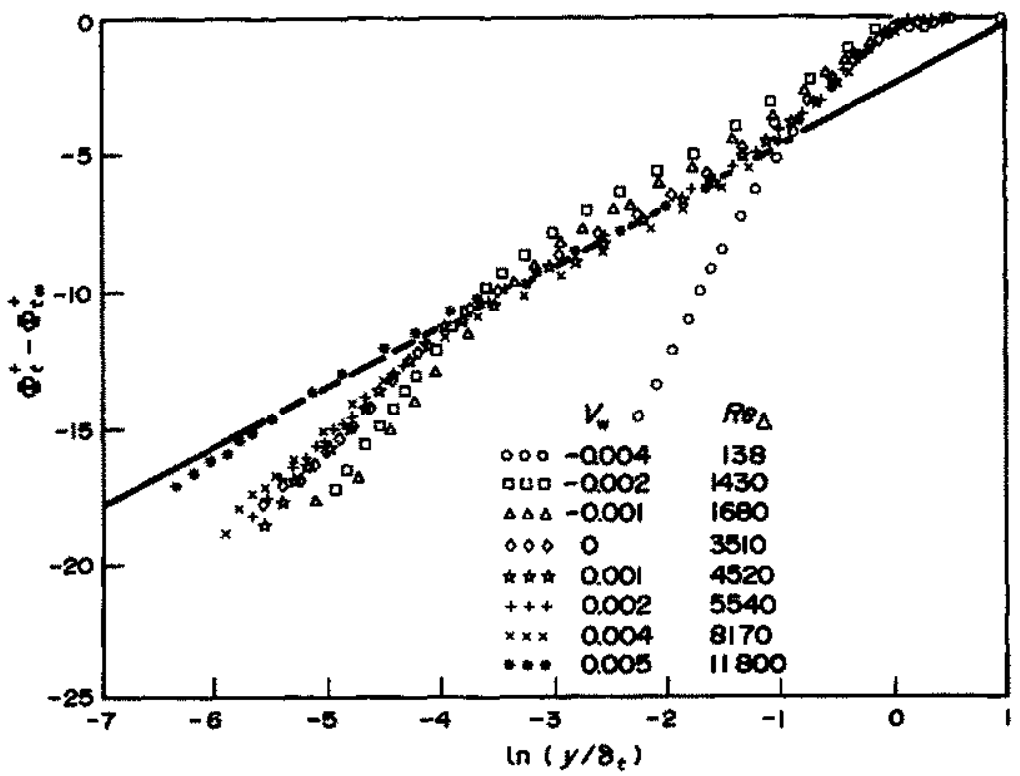

FIG. 2. Temperature law of the wake for transpired flow. Data from Whitten et al. [6]. 


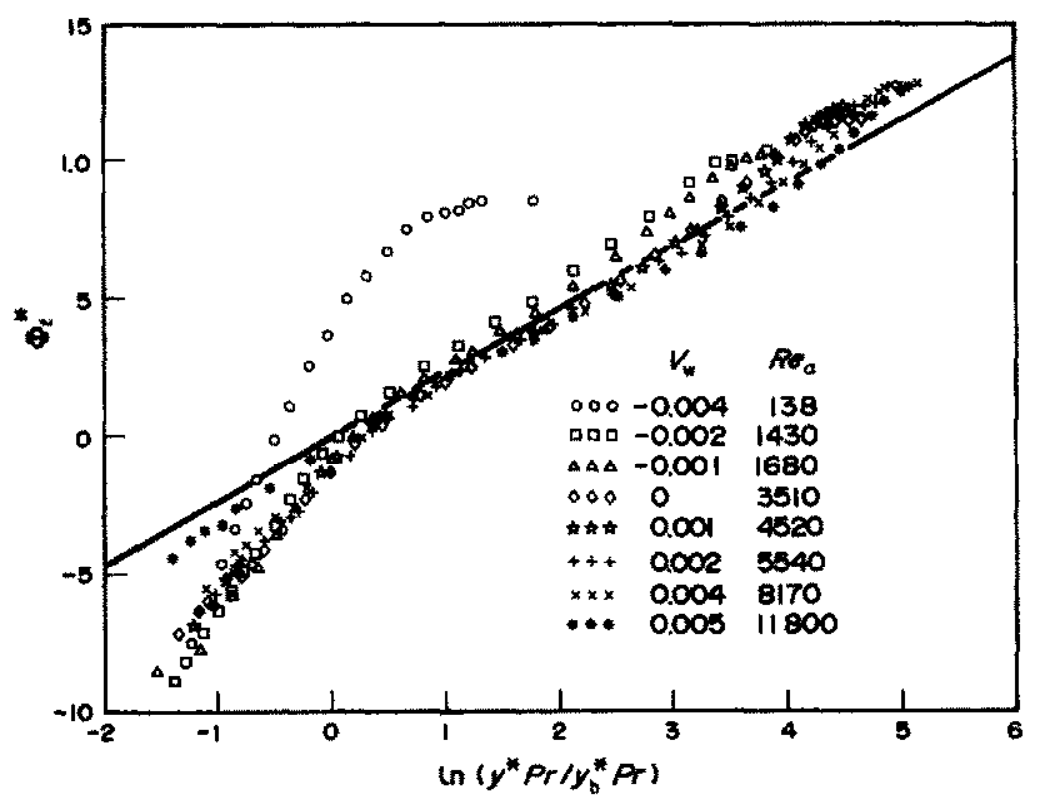

FIG. 3. Temperature law of the wall for transpired flow. Alternative approach. Data from Whitten et al. [6].

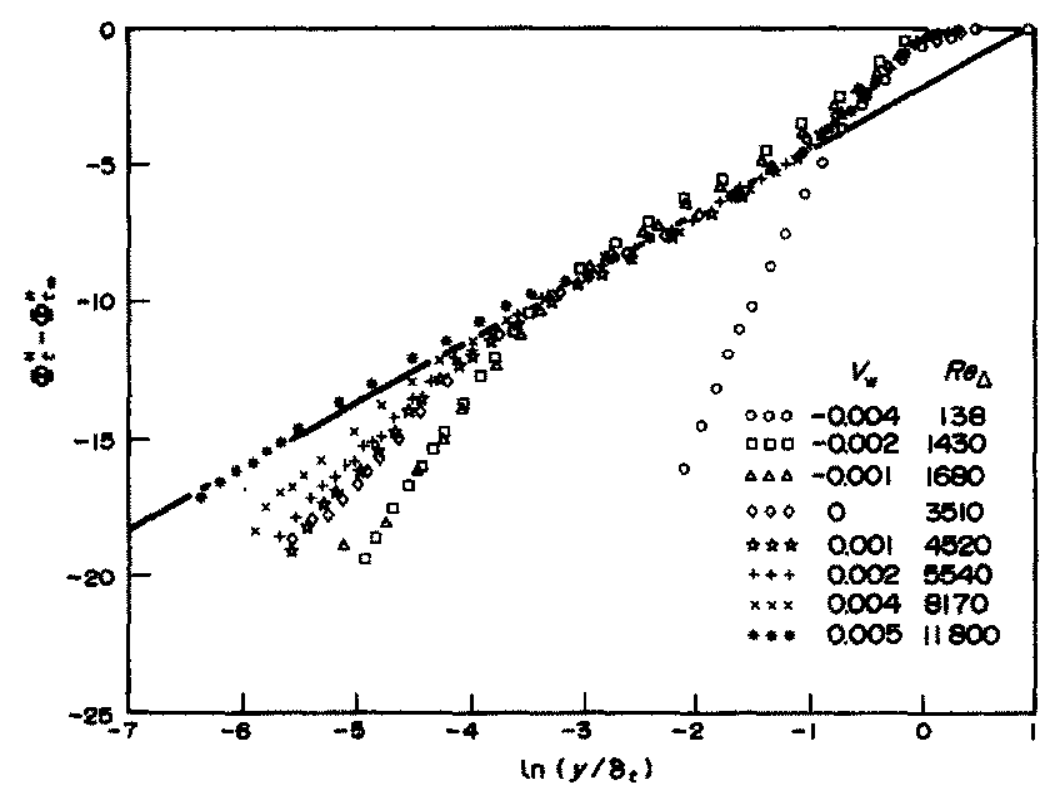

Flg. 4. Temperature law of the wake for transpired flow. Alternative approach. Data from Whitten $e t$ al. [6].

Of course application of equation (15) requires previous knowledge of $S t_{0}$. Here we took values of $S t_{0}$ from equation (IV-9) of ref. [6]. The present results are within 5\% accuracy for the very high injection rates, improving the previous results which are $15 \%$ accurate. If experimental correlations for $y_{b}^{+}$are used instead of equation (7) the results can be further improved.

\section{CONCLUSION}

In the present work, an analysis of the heat transfer problem for a transpired turbulent boundary fayer has been performed. We have proposed new expressions for the law of the wall and for the law of the wake that give a good account of the temperature profile behaviour and which can be used to derive Stanton number equations. The overall agreement of the theory with the experimental data is good for both the temperature and the Stanton number predictions. This agreement is not better because we have opted for using analytic expressions for the determination of $y_{b}^{+}$and $y_{b}^{*}$ instead of considering any sort of experimental correlations. We have used expression (7) since this keeps our procedure self-contained. Of particular note is the good accuracy in the predictions of $S t$ for high injection rates.

Although comparisons are made just for air flow, we expect the present formulation to hold for any type of fluid. In fact, as suggested by the solid surface results of refs. [10, 11], we expect equation (4) to hold for other types of fluid 
Table 1.

\begin{tabular}{cclccc}
\hline \multicolumn{1}{c}{$v_{w}$} & $R e_{\Delta}$ & $S t_{\text {exp }}[14]$ & $S t$, equation (11) & $S t$, equation (14) & $S t$, equation (15) \\
\hline$-2.5 \times 10^{-3}$ & $9.6 \times 10^{2}$ & $3.46 \times 10^{-3}$ & $3.730 \times 10^{-3}$ & $3.683 \times 10^{-3}$ & $3.559 \times 10^{-3}$ \\
-2.2 & $1.4 \times 10^{3}$ & 3.14 & 3.303 & 3.296 & 3.221 \\
-1.1 & $1.7 \times 10^{3}$ & 2.63 & 2.791 & 2.744 & 2.762 \\
0.0 & $3.5 \times 10^{3}$ & 1.95 & 1.955 & 1.903 & \\
0.0 & $4.7 \times 10^{3}$ & 1.82 & 1.831 & 1.783 & 1.455 \\
0.9 & $5.7 \times 10^{3}$ & 1.43 & 1.472 & 1.398 & 1.546 \\
1.0 & $4.5 \times 10^{3}$ & 1.52 & 1.565 & 1.475 & 1.129 \\
1.8 & $7.1 \times 10^{3}$ & 1.12 & 1.167 & 1.072 & 0.59 \\
1.9 & $5.5 \times 10^{3}$ & 1.23 & 1.238 & 1.124 & 0.640 \\
3.7 & $1.1 \times 10^{4}$ & 0.66 & 0.658 & 0.560 & 0.399 \\
3.9 & $8.2 \times 10^{3}$ & 0.69 & 0.720 & 0.604 & 0.441 \\
4.8 & $1.2 \times 10^{4}$ & 0.50 & 0.476 & 0.380 & \\
5.0 & $9.4 \times 10^{3}$ & 0.53 & 0.518 & &
\end{tabular}

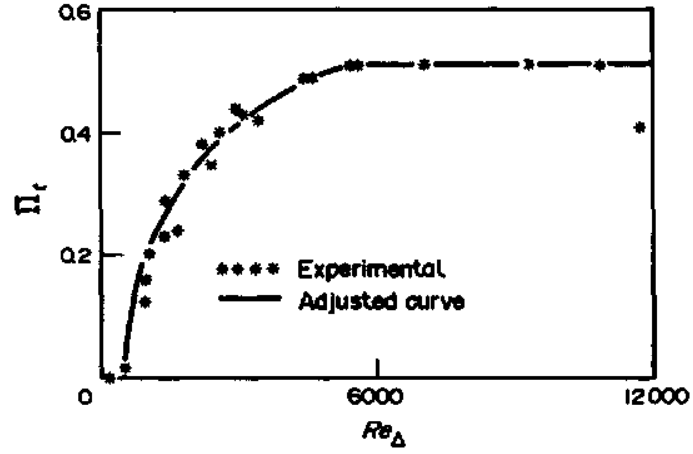

FIG. 5. Variation of $\Pi_{t}$ with $R e_{\Delta}$. Data from Whitten et al. [6] and Blackwell et al. [7].

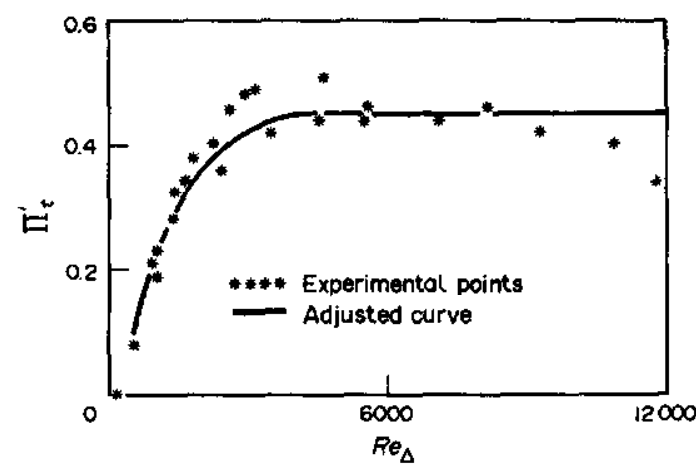

FIG. 6. Variation of $\Pi_{i}^{\prime}$ with $R e_{\mathrm{A}}$. Data of Whitten et al. [6] and Blackwell et al. [7].

only if the level of the logarithmic curve is made to vary appropriately with Pr. Since far away from the wall the convective effects are dominant, we expect to obtain an extension of equation (4) to the defect layer by adding Coles' function to its right-hand side.

Acknowledgements-The authors are grateful to FAPERJ, which financially supported the present work through grant No. E-29/170.358/88. MAFM is also grateful to CAPES for providing him with a scholarship during part of the execution of the work. Part of the references were kindly provided by Dr L. C. Squire and by Dr A. F. Orlando.

\section{REFERENCES}

1. R. J. Moffat and W. M. Kays, The turbulent boundary layer on a porous plate: experimental heat transfer with uniform blowing and suction, Int. J. Heat Mass Transfer 11, 1547-1566 (1968).

2. W. M. Kays and R. J. Moffat, The behavior of transpired turbulent boundary layers, Stanford University Report No. HMT-20 (1975).

3. T. N. Stevenson, A law of the wall for turbulent boundary layers with suction or injection, Cranfield College of Aero. Report No. 166 (1963).

4. T. N. Stevenson, A modified velocity defect law for turbulent boundary layers with injection, Cranfield College of Aero. Report No. 170 (1963).

5. R. L. Simpson, Characteristics of turbulent boundary layers at low Reynolds number with and without transpiration, J. Fluid Mech. 42, 769-802 (1970).

6. D. G. Whitten, W. M. Kays and R. J. Moffat, The turbulent boundary layer on a porous plate: experimental heat transfer with variable suction, blowing and surface temperature, Stanford University Report No. HMT-3 (1967)

7. B. F. Blackwell, W. M. Kays and R. J. Moffat, The turbulent boundary layer on a porous plate: an experimental study of the heat transfer behavior with adverse pressure gradients, Stanford University Report No. HMT-16 (1972).

8. A.P. Silva-Freire, An asymptotic solution for transpired incompressible turbulent boundary layers, Int. J. Heat Mass Transfer 31, 1011-1021 (1988).

9. R. A. Gowen and J. W. Smith, Turbulent heat transfer from smooth and rough surfaces, Int. J. Heat Mass Transfer 11, 1656-1673 (1968).

10. B. A. Kader and A. M. Yaglom, Heat and mass transfer laws for fully turbulent wall flows, Int. J. Heat Mass Transfer 15, 2329-2351 (1972).

11. B. A. Kader, Temperature and concentration profiles in fully turbulent boundary layers, Int. J. Heat Mass Transfer 24, 1541-1544 (1981).

12. D. B. Spalding, A standard formulation of the steady convective mass transfer problem, Int. J. Heat Mass Transfer 1, 192-207 (1960).

13. M. F. Blair, Influence of free-stream turbulence in turbulent boundary layer heat transfer and mean profile development, Part I - Experimental data, ASME J. Heat Transfer 105, 33-40 (1983).

14. M. F. Blair, Influence of free-stream turbulence in turbulent boundary layer heat transfer and mean profile development, Part II-Analysis of results, ASME J. Heat Transfer 105, 41-47 (1983). 